\title{
Defect solitons supported by nonlocal PT symmetric superlattices
}

\author{
Sumei $\mathrm{Hu}^{1,2}$, Daquan $\mathrm{Lu}^{1}$, Xuekai $\mathrm{Ma}^{1}$, Qi Guo ${ }^{1}$, and Wei $\mathrm{Hu}^{1, *}$ \\ ${ }^{1}$ Laboratory of Nanophotonic Functional Materials and Devices, \\ South China Normal University, Guangzhou 510631, P. R. China \\ ${ }^{2}$ Department of Physics, Guangdong University of Petrochemical Technology, Maoming 525000, P. R. China and \\ *Corresponding author: huwei@scnu.edu.cn
}

\begin{abstract}
The existence and stability of defect solitons supported by parity-time (PT) symmetric superlattices with nonlocal nonlinearity are investigated. In the semi-infinite gap, in-phase solitons are found to exist stably for positive or zero defects, but can not exist in the presence of negative defects with strong nonlocality. In the first gap, out-of-phase solitons are stable for positive or zero defects, whereas in-phase solitons are stable for negative defects. The dependence of soliton stabilities on modulation depth of the PT potentials is studied. It is interesting that solitons can exist stably for positive and zero defects when the PT potentials are above the phase transition points.
\end{abstract}

PACS numbers: $42.25 . \mathrm{Bs}, 42.65 . \mathrm{Tg}, 11.30 . \mathrm{Er}$

\section{INTRODUCTION}

Over the last few years, people have paid much attention to parity-time (PT) symmetric systems [1 9]. Bender et al have shown that a family of Hamiltonian with PT symmetry can still exhibit entirely real spectra, despite the non-Hermitian Hamiltonian [10]. PT symmetry requires that the complex potential satisfies $V(x)=V^{*}(-x)$. For a PT symmetric Hamiltonian, there exists a critical threshold above which its eigenvalues are not real but become complex, and the system undergoes a phase transition because of the spontaneous PT symmetry breaking [10]. Quite recently the notion of PT symmetry was introduced within the framework of optics [1]. This suggestion was based on judicious designs that involve both optical gain/loss regions and the process of index guiding [1, 5, 11]. Unusual properties such as unidirectional invisibility 12 and nonlinear switching structures [13, 14] have been found in PT symmetric structures. PT symmetry breaking in synthetic optical systems has been observed experimentally in semiconductor heterostructure [5], photorefractive crystals [6], and $L R C$ circuits [7].

Soliton phenomena in optical lattices and superlattices have attracted considerable attention [15-21]. Optical superlattices can be considered as regular lattices hosting a periodic chain of defects. Superlattices are fascinating because the structures exhibit collective properties not shared by either constituent, and these properties can be controlled through variation of the structural parameters [22]. Defect solitons in PT symmetric local lattices and superlattices have been studied and stable solitons are not found in the first gap for positive or zero defects [23, 24]. However, defect solitons in PT symmetric superlattices with nonlocal nonlinearity have not been studied. It is noteworthy that the nonlinearity in photorefractive media, in which Rüter et al have observed the PT symmetric wave propagation [6], is nonlocal nonlinearity [2527]. Nonlocality plays an important role in many areas of nonlinear physics [28], drastically modifies the properties of solitons and improves the soliton stability [28 30$]$.
Therefore it is worthy to study the properties of solitons in PT symmetric optical superlattices with nonlocal nonlinearity.

In this paper, we study defect solitons supported by the PT symmetric superlattices in nonlocal nonlinear media. We find that the nonlocality expands stability ranges of solitons, especially in the first gap. Both out-of-phase and in-phase solitons can be stable in the first gap for positive, zero, and negative defects. In-phase solitons can exist stably in the semi-infinite gap for positive or zero defects, but exist unstably for negative defects. When the modulation depth of the PT potentials is small, solitons can exist stably for positive and zero defects, even if the PT potentials are above the phase transition points.

\section{MODEL}

We consider the propagation of light beam in PT symmetric defective superlattices with Kerr-type nonlocal nonlinearity. The evolution of complex amplitude $U$ of the light fields can be described by following dimensionless nonlinear Schrödinger equation,

$$
\begin{gathered}
i \frac{\partial U}{\partial z}+\frac{\partial^{2} U}{\partial x^{2}}+[V(x)+i W(x)] U+n U=0 \\
n-d \frac{\partial^{2} n}{\partial x^{2}}=|U|^{2}
\end{gathered}
$$

where $x$ and $z$ are the transverse and longitudinal coordinates, respectively, $n$ is the nonlinear refractive-index change, $d$ stands for the degree of nonlocality of the nonlinear response. This type of nonlinear response with a finite region of nonlocality exists in many real physical systems, for instance, the photorefractive crystals used in the experimental observation of PT symmetry in optics [6]. When $d \rightarrow 0$, Eq. (2) reduces to $n=|U|^{2}$, and above equations reduce to the local case. $V(x)$ and $W(x)$ are the real and imaginary parts of the PT symmetric defective superlattices, respectively, which are assumed in this 
paper as

$$
\begin{aligned}
V(x) & =V_{0}\left[\epsilon_{1} \cos ^{2}(x)+\left(1-\epsilon_{1}\right) \sin ^{2}(2 x)\right] \\
& \times\left[1+\epsilon \exp \left(-x^{8} / 128\right)\right] \\
W(x) & =W_{0} \sin (2 x)
\end{aligned}
$$

Here $\epsilon$ and $\epsilon_{1}$ represent the strength of the defect and the modulation parameter of superlattice, respectively. When $0.1 \leq \epsilon_{1} \leq 0.7$, Eq. (3) has the superlattice shape 15. Without losing of generality, we take $\epsilon_{1}=0.5$ throughout the paper. The parameters $V_{0}$ and $W_{0}$ represent the modulation depth of the real and imaginary parts of the PT symmetric potentials, respectively.
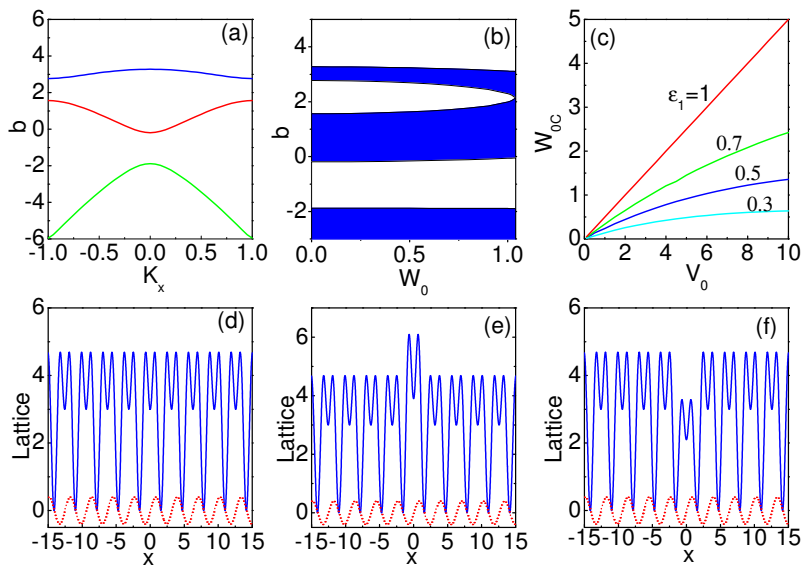

FIG. 1. (Color online) (a) Band structure of the superlattice with $V_{0}=6, W_{0}=0.4$ and $\epsilon_{1}=0.5$. (b) Band structure of the superlattice with different $W_{0}$ values, where $V_{0}=6$ and $\epsilon_{1}=0.5$. (c) The phase transition point $W_{0 C}$ versus $V_{0}$ for different $\epsilon_{1}$ values. (d)-(f) Lattice intensity profiles for the superlattice with defects (d) $\epsilon=0.3$, (e) $\epsilon=0$, and (f) $\epsilon=-0.3$ Blue solid lines and red dotted lines represent the real and imaginary parts, respectively.

The PT symmetric superlattices in Eq. (1) have a Bloch band structure when $\epsilon=0$ and $n=0$. The Bloch band structure obtained by the plane wave expansion method for $\epsilon_{1}=0.5, V_{0}=6$ and $W_{0}=0.4$ is shown in Fig 1(a). One can see that the region of the semiinfinite gap is $b>3.27$, and the first and second gaps are $1.56<b<2.77$ and $-1.88<b<-0.19$, respectively, where $b$ is the propagation constant. Figure $1(\mathrm{~b})$ shows the Bloch band structure for $V_{0}=6$ with different $W_{0}$ values. The region of the first gap decreases with increasing $W_{0}$. The first gap disappears at $W_{0}=1.035$, which is the phase transition point $W_{0 C}$. When the system is above the phase transition point, i.e. $W_{0}>1.035$, the band structure becomes complex. We find that, for the PT symmetric superlattices, $W_{0 C}$ changes with $V_{0}$ nonlinearly, as shown in Fig. 1(c). However, for the ordinary lattices $\left(\epsilon_{1}=1\right)$, the $W_{0 C}$ increases linearly with increasing $V_{0}$, i.e, $W_{0 c} / V_{0}=0.5$ [1, 2]. In this paper, we study the PT symmetric superlattices below or above the phase transition points, respectively. Figures 1(d)-1(f) show the intensity distributions of the PT superlattice potentials with the strength of defects $\epsilon=0, \epsilon=0.3$ and $\epsilon=-0.3$, respectively. $\epsilon=0$ corresponds to the uniform superlattice.

We search for stationary solutions to Eqs. (1) and (2) in the form $U=f(x) \exp (i b z)$, where $f(x)$ is a complex function and satisfies equations,

$$
\begin{gathered}
b f=\frac{\partial^{2} f}{\partial x^{2}}+[V(x)+i W(x)] f+n f, \\
n-d \frac{\partial^{2} n}{\partial x^{2}}=|f|^{2} .
\end{gathered}
$$

The solutions of defect solitons are gotten numerically from Eqs. (5) and (6) by the modified squared-operator method 31] and shown in the next section. To elucidate the stability of defect solitons, we search for the perturbed solutions to Eqs. (10) and (2) in the form $U(x, z)=[f(x)+u(x, z)+i v(x, z)] \exp (i b z)$, where the real $[u(x, z)]$ and imaginary $[v(x, z)]$ parts of the perturbation can grow with a complex rate $\delta$ upon propagation, i.e. $u(x, z)=u(x) \exp (i \delta z)$ and $v(x, z)=v(x) \exp (i \delta z)$. Substituting the perturbed solution into Eq. (1) and linearization of it around the stationary solution $f(x)$ yields the eigenvalue problem

$$
\begin{aligned}
\delta v= & \frac{\partial^{2} u}{\partial x^{2}}+(n-b) u+(V u-W v) \\
& +\operatorname{Re}[f(x)] \int_{-\infty}^{\infty} 2 G(x-\xi) u(\xi) \operatorname{Re}[f(\xi)] \mathrm{d} \xi \\
& +\operatorname{Re}[f(x)] \int_{-\infty}^{\infty} 2 G(x-\xi) \operatorname{Im}[f(\xi)] v(\xi) \mathrm{d} \xi \\
\delta u= & -\frac{\partial^{2} v}{\partial x^{2}}-(n-b) v-(W u+V v) \\
& -\operatorname{Im}[f(x)] \int_{-\infty}^{\infty} 2 G(x-\xi) \operatorname{Im}[f(\xi)] v(\xi) \mathrm{d} \xi \\
& -\operatorname{Im}[f(x)] \int_{-\infty}^{\infty} 2 G(x-\xi) \operatorname{Re}[f(\xi)] u(\xi) \mathrm{d} \xi .
\end{aligned}
$$

Here $G(x)=[1 /(2 \sqrt{d})] \exp (-|x| / \sqrt{d})$ is the response function of the nonlocal media. Above eigenvalue problem is solved numerically by the original-operator iteration method[32] to find the maximum value of $\operatorname{Re}(\delta)$. If $\operatorname{Re}(\delta)>0$, solitons are unstable. Otherwise, they are stable.

\section{NUMERICAL RESULTS}

In the nonlocal PT symmetric defective superlattices, we find two types of defect solitons. The first type is in-phase soliton, whose real part of optical fields is symmetric and the imaginary part is antisymmetric. The other type is out-of-phase soliton, whose real part is antisymmetric and the imaginary part is symmetric. It is noteworthy that if $U=f(x) \exp (i b z)$ is a solution to Eqs. 1 and 2, we also have a series of solution, i.e. $U=f(x) e^{i \theta} \exp (i b z)$, where $\theta$ is an arbitrary initial phase. We know that $f(x)$ and $f(x) e^{i \theta}$ represent the same physical soliton despite the initial phase, but their parities are uncertain. Due to PT symmetry, one can 




FIG. 2. (Color online) (a) Soliton power $P$ versus propagation constant $b$; (b) Cutoff point $b_{c}$ versus the depth of defects. (c) Unstable growth rate $\operatorname{Re}(\delta)$ versus propagation constant $b$ for out-of-phase solitons in the first gap. For all cases $V_{0}=6$, $W_{0}=0.4$, and $\epsilon=0.3$ except for (b).
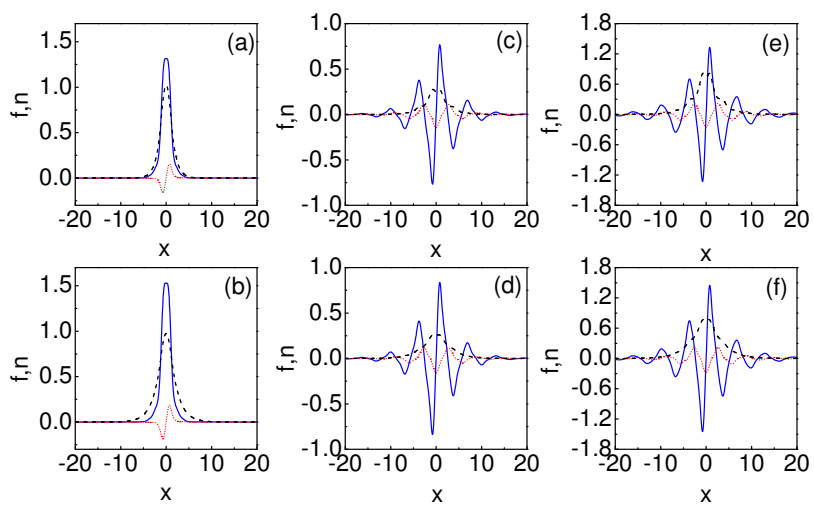

FIG. 3. (Color online) The complex fields (solid blue: real part; dotted red: imaginary part) and refractive-index changes (dashed black) for in-phase solitons in the semiinfinite gap at (a) $b=5, d=1$, and (b) $b=5, d=3$, and for out-of-phase solitons in the first gap at (c) $b=2.2$, $d=1$, (d) $b=2.2, d=3$, (e) $b=2.6, d=1$, (f) $b=2.6, d=3$, respectively. For all cases $\epsilon=0.3, V_{0}=6$ and $W_{0}=0.4$.

find a proper initial phase to guarantee that both the real and imaginary parts of solitons have fixed parity (odd or even). When we use a real function as the initial trial in the iteration program solving Eqs. (5) and (6), the final convergency solution has a certain parity and its real part is always bigger than its imaginary part. Then we can distinguish the in-phase and out-of-phase solitons in this occasion. We find that in the first gap, out-of-phase solitons can exist stably for positive and zero defects, whereas in-phase solitons can exist stably for negative defects. In the semi-infinite gap, we find in-phase solitons for both positive, zero, and negative defects.

For positive defects, we assume $\epsilon=0.3$ and the results are shown in Figs. 2, 4. We find in-phase solitons in the semi-infinite gap and out-of-phase solitons in the first gap, respectively. Figure 2(a) shows that the power of solitons [defined as $P=\int_{-\infty}^{+\infty}|f(x)|^{2} \mathrm{~d} x$ ] for both inphase and out-of-phase solitons increases almost linearly as increasing of propagation constant. There exists a cutoff point of propagation constant, below which the defect solitons vanish. The propagation constants of the cutoff points do not depend on the nonlocality degree. This feature is similar to the case of traditional uniform lattices
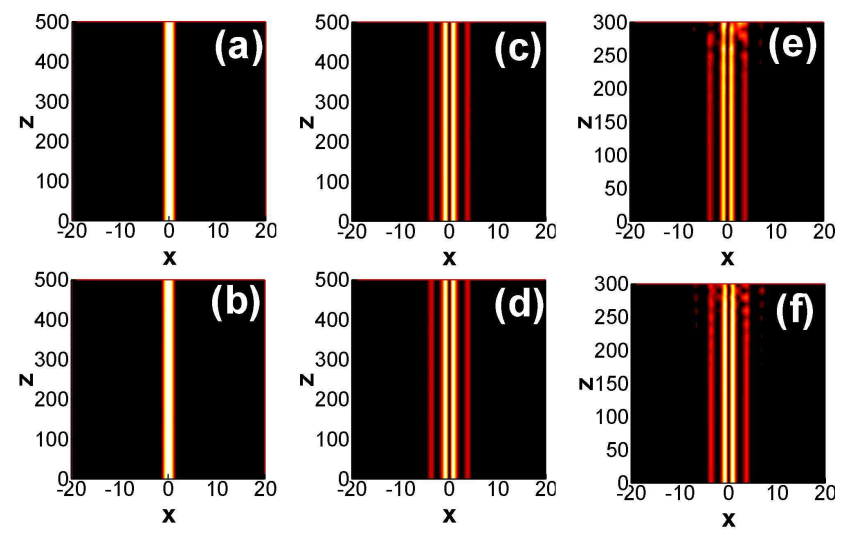

FIG. 4. (Color online) (a)-(f) Evolutions of defect solitons corresponding to Figs. 3(a)- 3(f), respectively.

in nonlocal media [29]. The propagation constants of the cutoff points for both in-phase and out-of-phase solitons increase with increasing the depth of positive defects, as shown in Fig. 2(b). When $\epsilon>0.725$, the out-of-phase solitons can not exist in the first gap.

We find that in-phase solitons are stable in the whole regime where solitons exist in the semi-infinite gap when $W_{0}=0.4$, like its counterparts in local PT lattices. Outof-phase solitons are found to exist stably for the low propagation constants in the first gap, as shown in Fig. 2(c). The stability regions of defect solitons decrease with increasing $W_{0}$. In-phase solitons in the semi-infinite gap become unstable for large propagation when $W_{0}>0.46$ and their stability region vanishes totally as $W_{0}>0.58$. Out-of-phase solitons become unstable in the whole first gap when $W_{0}>0.53$. For comparison, out-of-phase solitons in the first gap do not exist in local PT symmetric defective superlattices with positive defects [24]. It shows that the nonlocality expands the existing and stable ranges of solitons.

The field distributions of defect solitons are shown in Figs. 3(a)-3(f) for different nonlocal degrees and propagation constants, which correspond to the cases represented by circls in Fig. 2(a). Figures 3(a) and 3(b) show that in-phase solitons exist in the semi-infinite gap, while solitons in the first gap are out-of-phase as shown in Figs. 3( (c)-3(f). As the nonlocality degree increases, soliton power increases but the shape of their field distribution changes very little. The propagations of solitons are also simulated based on Eqs. (11) and (2), and $1 \%$ random-noise perturbations are added into the initial input to verify the results of the linear stability analysis. The propagations corresponding to solitons in Figs. 3(a)-3(f) are shown in Fig. 4(a)-4(f). We can see that in-phase solitons are stable in the semi-infinite gap and out-of-phase solitons are stable in the first gap for the low propagation constants.

Figures 5177 show the results for zero defects $(\epsilon=0)$. The properties of in-phase solitons in the semi-infinite gap and out-of-phase solitons in the first gap for zero 

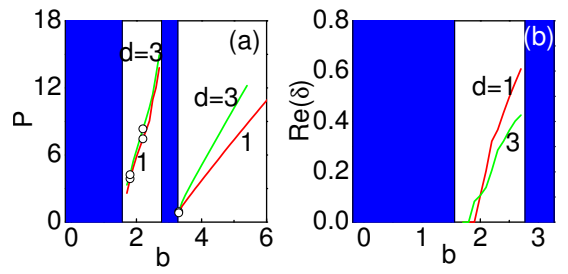

FIG. 5. (Color online) (a) Soliton Power $P$ versus propagation constant $b$ for different value of $d$. (b) Unstable growth rate $R e(\delta)$ versus propagation constant $b$ for out-of-phase solitons in the first gap. For all cases $V_{0}=6, W_{0}=0.4$, and $\epsilon=0.0$


FIG. 6. (Color online) The complex fields (solid blue: real part; dotted red: imaginary part) and refractive-index changes (dashed black) for in-phase solitons in the semiinfinite gap at (a) $b=3.3, d=1$ and (b) $b=3.3, d=3$, and for out-of-phase solitons in the first gap at (c) $b=1.8$, $d=1,(\mathrm{~d}) b=1.8, d=3$, (e) $b=2.2, d=1$ and (f) $b=2.2$, $d=3$, respectively.

defects are almost the same as those for positive defects, except the positions of the cutoff points. From Fig. 5)(a) we can see that the cutoff points approach the edge of the gap, so in-phase solitons are stable in the whole semi-infinite gap when $W_{0}=0.4$. For comparison, the local defect solitons in the semi-infinite gap are unsta-
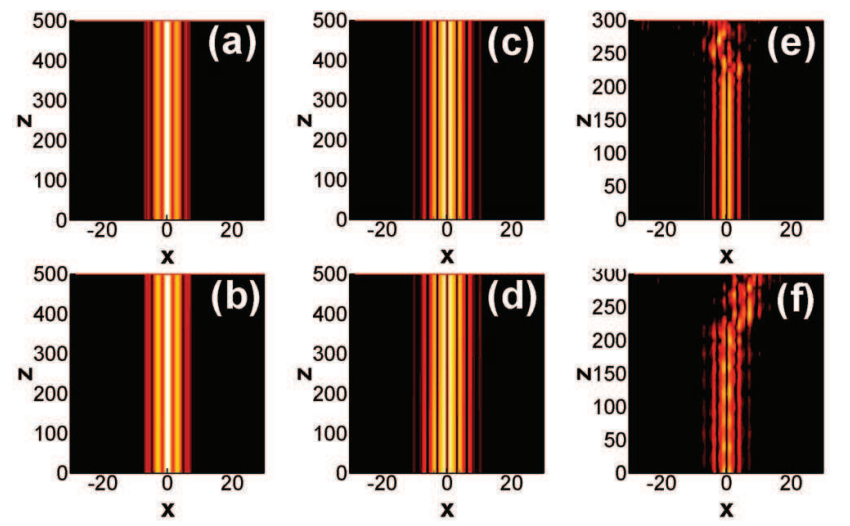

FIG. 7. (Color online) (a)-(f): Evolutions of defect solitons corresponding to Figs. 5(c)- 5.(h), respectively.
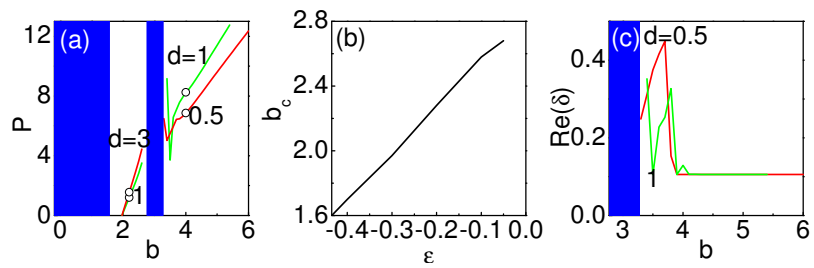

FIG. 8. (Color online) (a) Soliton Power $P$ vs propagation constant $b$ for in-phase solitons in the semi-infinite gap and the first gap. (b)Cutoff point $b_{c}$ versus the depth of defects. (c) Unstable growth rate $R e(\delta)$ versus propagation constant $b$ for the in-phase solitons in the semi-infinite gap. For all cases $V_{0}=6, W_{0}=0.4$, and $\epsilon=-0.3$ except for (b).
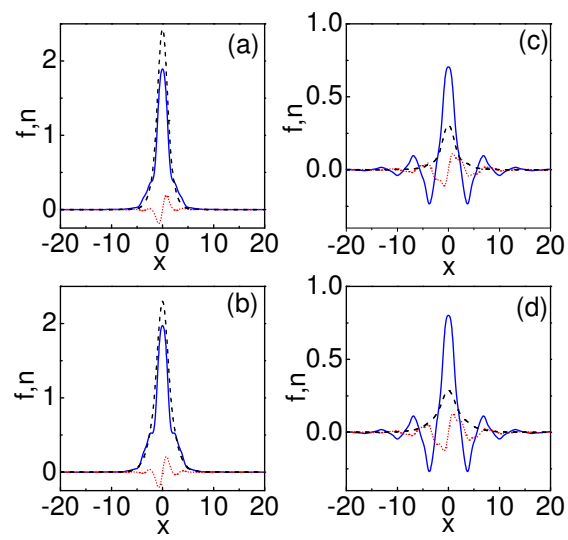

FIG. 9. (Color online) (a)-(d) The complex fields (solid blue: real part; dotted red: imaginary part) and refractive-index changes (dashed black) for in-phase solitons in the semiinfinite gap at(a) $b=4, d=0.5$ and (b) $b=4, d=1$, or in the first gap at (c) $b=2.2, d=0.5$ and (d) $b=2.2, d=1$, respectively.

ble near the edge of the gap 24]. For nonlocal superlattices, we find that in-phase solitons are always stable when $W_{0}<0.48$. As increasing $W_{0}$, the stable region begins to shrink, and in-phase solitons are unstable in the whole semi-infinite gap when $W_{0}>0.76$.

Figure 5 (b) is the perturbation growth rate $\operatorname{Re}(\delta)$ versus propagation constant for out-of-phase solitons in the first gap with different nonlocality degree when $W_{0}=0.4$. We can see that out-of-phase solitons are stable for the low propagation constant. As increasing $W_{0}$, the stability region of out-of-phase solitons vanishes after $W_{0}>0.51$.

The examples of in-phase solitons in the semi-infinite gap and out-of-phase solitons in the first gap for zero defects are shown in Figs. 6(a)-6(f), and the corresponding propagations are shown in Fig. 7. We can see that inphase solitons in the semi-infinite gap have more peaks than those for positive defect. Figure 7 shows that inphase solitons are stable at the edge of the gap whereas out-of-phase solitons are stable with low propagation constants. However, out-of-phase solitons in local PT symmetric defective superlattices do not exist in the first gap for zero defects [24]. 

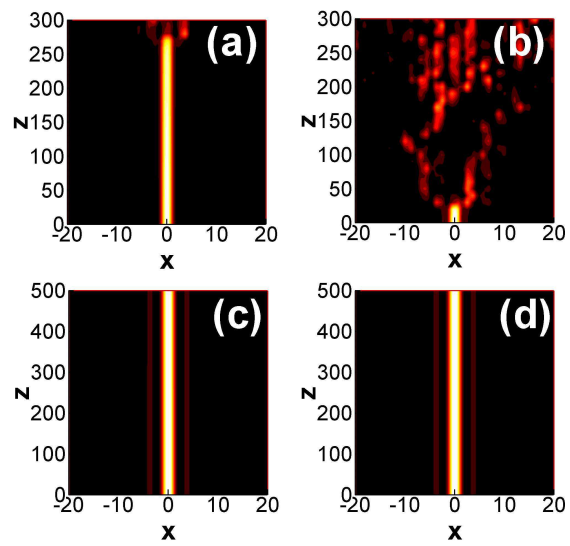

FIG. 10. (Color online) (a)-(d): Evolutions of defect solitons corresponding to Figs. 8(a)- 8(d), respectively.

For negative defects, we assume $\epsilon=-0.3$ and the results are shown in Figs. 8, 10, We find that in-phase solitons in the semi-infinite gap exist only for a weak nonlocality degree $(d<1.2)$, and our numerical program can not find any stationary solution for $d>1.2$. The linear stability analysis shows that in-phase solitons in the semi-infinite gap are unstable in their whole existence regions, as shown in Fig. 8(c). In the first gap, differing from positive and zero defects, in-phase solitons are found and stable in their whole existence regions when $W_{0}<0.56$. As increasing $W_{0}$, in-phase solitons in the first gap become unstable for the large propagation constant when $W_{0}<0.78$. Solitons are all unstable after $W_{0}>0.78$.

We can also see that there exists a cutoff point of propagation constant above which in-phase solitons exist. This feature is similar to the case for positive and zero defects. Figure 8(b) shows that the cutoff point shifts toward the lower propagation constant with increasing the depth of negative defects, and the existence region of in-phase solitons in the first gap increases too. When $\epsilon=-0.435$, the cutoff point arrives at the edge of the gap and then in-phase solitons exist stably in the whole first gap for $W_{0}=0.4$.

The examples of in-phase solitons in the semi-infinite gap and the first gap are shown in Figs. 9(a)-9(d), and the corresponding propagations are shown in Figs. 10(a)10(d). One can see that in-phase solitons are unstable in the semi-infinite gap whereas they are stable in the first gap.

Finally, we study the case of PT symmetric superlattices above the phase transition point. We find that inphase solitons for positive and zero defects can exist stably even if $\mathrm{PT}$ potentials are above the phase transition points when the value of $V_{0}$ is small. We take $V_{0}=0.2$ and the phase transition point $W_{0 c}=0.05$, as shown in the fig. 1(c). Figures 11(a) and 11(b) show the real and imaginary parts of bandgap structures for $V_{0}=0.2$ and $W_{0}=0.15$, respectively. One can see that the corresponding band diagram is partially complex. Figures.
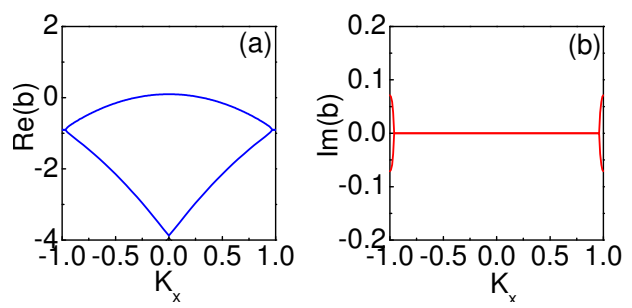

FIG. 11. (a) Real and (b) imaginary parts of the bandgap structures for the superlattice with $\epsilon_{1}=0.5, V_{0}=0.2$ and $W_{0}=0.15$, respectively.
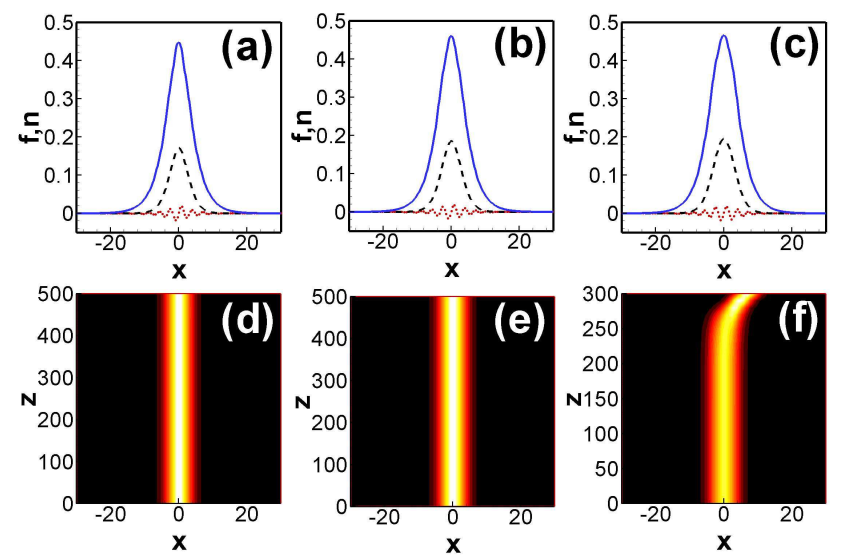

FIG. 12. (Color online) The complex fields (solid blue: real part; dotted red: imaginary part) and refractive-index changes (dashed black) of solitons for (a) positive defect $\epsilon=0.3$, (b) zero defect $\epsilon=0$, and (c) negative defect $\epsilon=-0.3$. (d)-(f) Evolutions of defect solitons corresponding to these solitons in (a)-(c), respectively. For all cases $V_{0}=0.2, W_{0}=0.15, b=0.2$, and $d=1$.

12(a)-12 (c) show the examples of solitons above the phase transition points for positive $(\epsilon=0.3)$, zero $(\epsilon=0)$, and negative defects $(\epsilon=-0.3)$, respectively. Figures [12(d)12(f) show the corresponding propagations of those solitons. Supported by the nonlocal nonlinearity $(d=1)$, defect solitons for positive and zero defects are stable when $W_{0}$ is above the phase transition point, whereas they are unstable for negative defects. It is noted that the imaginary parts of the complex fields for solitons shown in Fig. 12 are still very small comparing with their real parts, although the PT potentials are above the phase transition points.

\section{CONCLUSION}

We have studied the existence and stability of defect solitons supported by parity-time symmetric nonlocal superlattices. Unusual properties are found in this system. The nonlocality can drastically affect the existence and stability of defect solitons. For positive or zero defects, in-phase solitons can exist stably in the semi-infinite gap and out-of-phase solitons can exist stably in the first gap. 
For negative defects, in-phase solitons are stable in the first gap, but unstable in the semi-infinite gap for the weak nonlocality degree. The values of $W_{0}$ and $V_{0}$ affect the soliton stability strongly. When $V_{0}$ is large, defect solitons are unstable unless $W_{0}$ is far below the phase transition point $W_{0 C}$. However, when $V_{0}$ is small, the stable solitons can be found for positive and zero defects even if $W_{0}$ is above the phase transition points. These properties of defect solitons in PT symmetric nonlocal superlattices are obviously different from those in the lo- cal PT superlattices.

\section{ACKNOWLEDGMENTS}

This research was supported by the National Natural Science Foundation of China (Grant Nos. 10804033, 11174090, and 11174091).
[1] Z. H. Musslimani, K. G. Makris, R. El-Ganainy, and D.N. Christodoulides, Phys. Rev. Lett. 100, 030402(2008).

[2] K. G. Makris, R. El-Ganainy, D. N. Christodoulides, and Z. H. Musslimani, Phys. Rev. A 81, 063807(2010).

[3] O. Bendix, R. Fleischmann, T. Kottos, and B. Shapiro, J. Phys. A: Math. Theor. 43, 265305(2010).

[4] C. T. West, T. Kottos, and T. Prosen, Phys. Rev. Lett. 104, 054102(2010).

[5] A. Guo, G. J. Salamo, D. Duchesne, R. Morandotti, M. Volatier-Ravat, V. Aimez, G. A. Siviloglou, and D.N. Christodoulides, Phys. Rev. Lett. 103, 093902(2009).

[6] C. E. Ruter, K. G. Makris, R. El-Ganainy, D. N. Christodoulides, M. Segev and D. Kip, Nature Phys. 6, 192(2010).

[7] J. Schindler, Ang Li, M. C. Zheng, F. M. Ellis, and T. Kottos, Phys. Rev. A. 84,040101(R)(2011).

[8] F. Abdullaev, Y. V. Kartashov, V. V. Konotop, and D. A. Zezyulin, Phys. Rev. A 83,041805(2011).

[9] H. G. Li, Z. W. Shi, X. J. Jiang, and X. Zhu Opt. Lett. 36,3290(2011).

[10] C. M. Bender, D. C. Brody, and H. F. Jones, J. Math. Phys. 40,2201(1999).

[11] M. C. Zheng, D. N. Christodoulides, R. Fleischmann, and T. Kottos, Phys. Rev. A 82,010103(2010).

[12] Z. Lin, H. Ramezani, T. Eichelkraut, T. Kottos, H. Cao, and D. N. Christodoulides, Phys. Rev. Lett. 106,213901(2011).

[13] H. Ramezani, T. Kottos, R. El-Ganainy, and D.N. Christodoulides, Phys. Rev. A 82,043803(2010).

[14] A. A. Sukhorukov, Z. Y. Xu, and Y. S. Kivshar, Phys. Rev. A82,043818(2010).

[15] W. H. Chen, Y. J. He, and H. Z. Wang, Opt. Express 15,14498(2007).

[16] Z. Y. Zhang, and S. J. Xiong, Phys. Rev. B

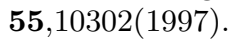

[17] K. N. Alekseev, G. P. Berman, D. K. Campbell, E. H. Cannon, and M. C. Cargo, Phys. Rev. B 54,10625(1996).

[18] M. A. Porter, P.G. Kevrekidis, R. Carretero-Gonzalez, and D.J. Frantzeskakis, Phys. Lett. A 352, 210(2006).

[19] M. Ghulinyan, C. J. Oton, Z. Gaburro, L. Pavesi, C. Toninelli, and D. S. Wiersma, Phys. Rev. Lett. 94,127401(2005).

[20] F. Dreisow, A. Szameit, M. Heinrich, T. Pertsch, S. Nolte, A. Tnnermann, and S. Longhi, Phys. Rev. Lett. 102, 076802(2009).

[21] X. Zhu, H. Wang, L. X. Zheng, H. G. Li, and Y. J. He, Opt. Lett. 36, 2680(2011).

[22] W. Chen, and D. L. Mills, Phys. Rev. Lett. 58, 160(1987).

[23] H. Wang and J. Wang, Opt. Express 19, 4030(2011).

[24] Z. E. Lu, and Z. M. Zhang, Opt. Express 19, 11457(2011).

[25] M. Segev, B. Crosignani, A. Yariv, and B. Fischer, Phys. Rev. Lett. 68, 923(1992).

[26] M. Segev, G. C. Valley, B. Crosignani, P. DiPorto, and A. Yariv, Phys. Rev. Lett. 73, 3211(1994).

[27] W. Krolikowski, M. Saffman, B. Luther-Davies, and C. Denz, Phys. Rev. Lett. 80, 3240(1998).

[28] A.W. Snyder and D. J. Mitchell, Science 276, 1538(1997).

[29] Z. Xu, Y. V. Kartashov, and L. Torner, Phys. Rev. Lett. 95, 113901(2005).

[30] D. Buccoliero, A. S. Desyatnikov, W. Krolikowski, and Y. S. Kivshar, Phys. Rev. Lett. 98, 053901(2007).

[31] J. Yang, and T. I. Lakoba, Stud. Appl. Math. 118, 153(2007).

[32] J. Yang, J. Comput. Phys. 227, 6862(2005). 